\title{
Clear-Cut Terms and Culture-Sensitive Characteristics of Distinctive Casual Sexual Relationships in Portuguese Emerging Adults
}

\author{
Maria-João Alvarez ${ }^{1}$ (D) . Cícero R. Pereira ${ }^{2}$ (i) . Cristina A. Godinho ${ }^{3,4}$. \\ Rita Luz ${ }^{1}$ (D)
}

Accepted: 29 March 2021 / Published online: 10 April 2021

(C) The Author(s), under exclusive licence to Springer Science+Business Media, LLC, part of Springer Nature 2021

\begin{abstract}
Casual sexual relationships (CSR) are increasingly common but limited empirical research has addressed their terminology and distinctive characteristics. This study sought to identify the most clear-cut terms and to consider how culture-sensitive characteristics distinguish casual sexual relationships among Portuguese emerging adults ( $N=262,18-29$ years old). We combined two qualitative studies - one by association and another by free recall - to ascertain the clarity of the terms, plus a quantitative study to further characterize and differentiate them. Participants were asked to match descriptions of CSRs with the respective terms by which they are known (Study 1). The same was investigated using an evocation task requesting that participants produce the terms by which the described CSRs are known (Study 2 ). Binary logistic regressions were performed to analyze the associations between encounters and labels chosen, taking the effect of gender and age into account. In the third study, participants rated the degree to which eight characteristics were present in the types of CSR, a simultaneous task that led to greater understanding of their descriptive and differentiating characteristics. Multivariate analysis of variance was used, with gender and age as covariates. We conclude that two types of CSR friends with benefits and one-night stand - and, to a lesser extent, making out, are understood and associated with consensual labels, also found by free-recall. These CSRs proved to be distinct, as they are understood as more different than similar in a set of psychoemotional, behavioral, and sexual characteristics.
\end{abstract}

Maria-João Alvarez

mjalvarez@psicologia.ulisboa.pt

1 Faculdade de Psicologia, CICPSI, Universidade de Lisboa, Lisboa, Portugal

2 Universidade Federal da Paraíba, João Pessoa, Brasil

3 Católica Research Centre for Psychological - Family and Social Wellbeing, Universidade Católica Portuguesa, Lisboa, Portugal

4 CIS-IUL, Instituto Universitário de Lisboa (ISCTE-IUL), Lisboa, Portugal 
Keywords Casual sexual relationships $\cdot$ Labels $\cdot$ Distinctiveness $\cdot$ Emerging adults

\section{Introduction}

Abundant evidence shows that sex researchers should not assume that participants subscribe to a particular definition of any sexual term (Randall \& Byers, 2003). However, limited empirical study has addressed the terminology, definitions, and characteristics of casual sexual relationships (CSR) (see Jonason, 2013; Wentland \& Reissing, 2014 for an exception). Different conceptualizations of "casual sex" have been found (e.g., Weaver \& Herold, 2000), with a myriad of relationships considered under this term (Claxton \& Dulmen, 2013), indicating that researchers must take this diversity into consideration and be clear in their definitions. In addition to the importance a clear definition has for improving the accuracy of research results and the psychosocial relevance of differentiating characteristics among CSR, knowledge about the most-used designations by participants has scientific relevance. Designations do not all have the same cognitive efficiency - that is, are not equally informative and differentiated, with those naturally chosen by individuals being easier to use for understanding and acting in a given situation (Rosch et al., 1976). Since the types of CSR found in the various studies are not the same (e.g., Nelson et al., 2011; Singer et al., 2006; Wentland \& Reissing, 2011), this may arise not only from idiosyncrasies in the samples, but also from sensitivity to the cultural environment where the encounters take place (Bogle, 2008), which underlines the relevance of studying CSR in different cultures.

Our approach is integrated in the general perspective of social constructionism, in which shared beliefs within a particular social group are at the root of the interpretation of reality, with sexuality created by culture (Delamater \& Hide, 1998). It is also informed by sexual script theory (Simon \& Gagnon, 1986) in its basic premise that sexual behavior is socially scripted. Cultural scenarios, the most abstract level of scripting, guide individuals according to the meanings that exist in communal life and demarcate the playing field of sexuality. Particularly in postparadigmatic societies, where diversity of behavior is more accepted, each social organization entails a great deal of variability, which manifests in different collective meanings (Wiederman, 2016).

There are numerous cultures in which casual sexual relationships are prevalent, having occurred at least once to $40-80 \%$ of young adults (Alvarez et al., 2019; Correa et al., 2017; Garcia et al., 2012, Wentland \& Reissing, 2014). We want to contribute so that the terminology and characteristics used in the studies accurately reflect participants' understanding of the concept. Based on the definitions, terms, and characteristics previously obtained for CSR known of or experienced (Alvarez et al., 2019), we sought to identify the most clear-cut terms and to note how culture-sensitive characteristics distinguish casual sexual relationships among Portuguese emerging adults. 


\section{Definitions and Terminology: The Need for a Shared Understanding of Concepts}

Consistency in response to questions that use sexuality-related terms requires that these are operationally defined, lest misunderstandings occur in communicating with participants or amongst researchers themselves. This lack of consensus has been found for various concepts such as "having sex" (Pitts \& Rahman, 2001; Richters \& Song, 1999), "sexual partners", and "sexual infidelity" (Alvarez \& Nogueira, 2008; Randall \& Byers, 2003), for which different individuals applied different behavioral criteria. For "sexual monogamy", the paradigm used by researchers showed an understanding - sexual exclusivity - very different from that of the layperson - emotional exclusivity (Swan \& Thompson, 2016).

"Casual sexual relationships" are usually considered non-committed sexual relationships, involving a range of sexual activities from kissing to intercourse, devoid of expectations of romantic attachment, regardless of how well participants know each other or of the duration of the relationship (Claxton \& Dulmen, 2013; Grello, Welsh, \& Harper, 2006; Hatfield, Hutchison, Bensman, Young, \& Rapson, 2012). This very inclusive operationalization means that "involvement in casual sex" can vary between $13 \%$ when defined as sexual relations occurring on the same day the partner was met and $73 \%$ when asking about partners with whom there was sexual involvement without penetrative sex (Weaver \& Herold, 2000). Apart from the fact that results will vary according to the definitions chosen, research has identified a variety of CSRs. Following the definitions by Wentland and Reissing (2014), in addition to the umbrella term hookup, these varieties range from a single sexual encounter (one night stand) (Regan \& Dreyer, 1999), to a continuous but impersonal, utilitarian relation (booty call) (Jonason, Li, \& Cason, 2009; Jonason, Li, \& Richardson, 2011), to a relation that is more personal but focused on sex (fuck buddies) (Weave et al., 2011; Wentland \& Reissing, 2011), to a relationship that is more personal and not exclusively focused on sex (friends with benefits) (Afifi \& Faulkner, 2000).

The breadth of this concept contributes to disparate interpretations not only among participants, but also among researchers, depending on the criteria that they use, organized mainly around sexual behavior, level of intimacy, and length of the relationship (Claxton \& Dulmen, 2013; Weaver \& Herold, 2000; Wentland $\&$ Reissing, 2014). However, the definitional issues related to CSR are considered to largely stem from the variety of terms used by researchers themselves (Wentland \& Reissing, 2014), with a tendency to neglect that the accuracy of each term is very dependent on participants' perspectives. Besides the variations in participants' interpretations of CSRs that result from overly-broad or nonexistent definitions of casual sex, participants may interpret these terms differently due to their own conceptualizations of those relationships. If the first can be addressed in some way by the presentation of an operational definition, the way in which CSRs are conceptualized must be ascertained in communication with participants, with this mindset taken into account during the research. Failure to provide definitions of CSRs may decrease the accuracy of research results due to the risk of evaluating very different 
behaviors under the same label, limiting our understanding of the characteristics, causes, and consequences, while failure to take into consideration how participants conceptualize CSRs may result in lack of shared realities between researchers and young adults.

\section{The Role of the Clearest Cases}

Basic to social constructionism is the role of language and its categories, through which we make sense of the world, classify events and persons, and interpret experiences. Each society has a discourse about sex, its way of thinking and talking about what is involved in sexual expression (see Delamater \& Hide, 1998). In this specific cultural context, there are categories that individuals more naturally resort to when thinking, describing, and naming objects, as described in research on natural categorization processes (Rosch \& Mervis, 1975; Rosch et al., 1976). However, the prototypical perspective does not presuppose the universality of the content of the categories - rather, their content reflects the correlational structure of the world and individuals' levels of knowledge of this structure, with the expectation that the categories develop differently in different cultures.

The natural categories consist of diffuse sets structured around their clearest cases, a relation of family resemblance with the category prototype. However, there is a basic level of abstraction at which the categories are simultaneously more informative and more differentiated from the others (Rosch et al., 1976). For this reason, they display richer characteristics from a cognitive point of view, are cognitively more efficient, and are therefore used by individuals when referring to certain realities of the natural world, as well as to social situations (e.g., Cantor, 1980) and knowledge about events (Rifkin, 1985). Since sexual encounters are events, we anticipated a taxonomic organization associated with these encounters, establishing an analogy between the properties of the categories used to name and describe common objects and those used to classify situations of sexual interaction.

The existence of clearly-defined sexual encounters was considered important for the study of sexual scripts, which represent the sequence of stereotyped actions associated with specific sexual encounters, constituting a structure by which to understand and anticipate the behavior of others and for informing one's own actions (Simon \& Gagnon, 1986). The clearly-defined sexual encounters are important because they make it possible to establish a relationship between the example chosen as an event and the quality or usefulness of the script it initiates. This idea is based on studies that have shown that the events used as units for people's understanding of daily activities were also events for which there were scripts (Rosch, 1978). Thus, if there is a level of abstraction in which events seem to be placed, with their examples, that makes those events more appropriate to use in thinking about or naming them, and if there is a relationship between the characteristics of the actions used to characterize an event and the level of abstraction at which this event is presented, then the selection of emblematic sexual encounters proves to be a task of decisive consequence for the cognitive efficiency of knowledge activated by the script (Alvarez, 2005). 
With a sample of Portuguese emergent adults, employing the most-frequentlyused terms for CSR (Alvarez et al., 2019), we selected a procedure inspired by the prototypical perspective. Participants were asked to categorize the encounters in three steps: first in the smallest number of categories possible, then in a larger number, and, finally, in the largest number of categories (while still categorizing some encounters together), so as to mirror the three levels of the taxonomy proposed by Rosch and collaborators (1976) - superordinate, basic, and subordinate. After conducting multidimensional and cluster analyses for each step, judges evaluated the interpretation of the dimensions in which the encounters were organized and the most typical examples of the extreme of each dimension. The highest levels of agreement regarding the second step of categorization showed it to be more relevant for determining the present dimensions (sexual involvement, emotional involvement, and repetition) and encounters (one-night stand, friends with benefits, making out, going to bed, "enrolanço”, passing passion) (Brandão, 2019).

\section{Cultural Diversity and Studies of CSR}

Interest in less restrictive, short term involvement was associated with a phenomenon first identified on North American university campuses of a new norm favoring casual sexual contacts (Bogle, 2008). This is not just a behavior, but a climate where casual sex is expected, surrounded by a lot of alcohol that helps it to be meaningless, and has strict codes of conduct, which has been characterized as hookup culture (Heldman \& Wade, 2010; Garcia et al., 2012; Wade, 2017). The ecological nature of sexual behavior and its relationship to cultural acceptance of engaging in casual sex (Hatfield et al., 2012) imply that social and cultural variables are important in understanding young adults' intimate relationships, with an emphasis on the importance of studying these variables across multiple cultures (Claxton \& Dulmen, 2013). In fact, only $25 \%$ of the terms used were similar between North American samples and a Canadian sample (Nelson et al., 2011; Singer et al., 2006; Wentland \& Reissing, 2011), with the latter having $43 \%$ of its terms different from a Portuguese sample (Alvarez et al., 2019). These different designations may reflect different CSRs and characteristics across different cultures.

There are three main CSRs found and used in research: friends with benefits, onenight stand and booty call (Klipfel et al., 2014; Rodrigue et al., 2018; Wentland \& Reissing, 2011) to which fuck buddies is added (Wentland \& Reissing, 2011). In the first study of the designations used by a Portuguese sample to name the CSRs they know of or have experienced, the participants used two of these encounters - friends with benefits and one night stand - among the four most frequent, with "curte" (making out) and casual relationship joining them; these proved to be the most distinctive after a multidimensional analysis (Alvarez et al., 2019), three of which (those other than casual relationships) were found in later studies through a focus group (Brandão, 2019). Although the booty call is a type of casual encounter frequently referred to in several samples (e.g., Jonason et al., 2009), both the designation and its characteristics were rarely found in the Portuguese studies mentioned above. 
Apart from the task of ensuring that the terminology used accurately reflects participants' understanding of the concepts of CSR under investigation, our research was motivated by the fact that the distinctive features of CSRs have only recently been explored (Alvarez et al., 2019; Mongeau et al., 2013; Rodrigue et al., 2015; Wentland \& Reissing, 2011), and studies are still scarce. Although researchers believe that there is a diversity of CSRs, this presupposition has yet to be scientifically ascertained (Claxton \& Dulmen, 2013). Studies so far have mainly been of a descriptive nature, and the CSRs have been studied in split sets (Jonason, 2013). Therefore, researchers have yet to develop quantitative data that will help to establish potential differences and similarities among CSRs, or to include multiple CSRs in a single-study design.

\section{The Present Study}

Based on the triple argument that CSRs may be specific to a particular cultural environment, that their conceptualization may differ between researchers and participants, and that if CSRs consist of different instances, it is useful to know the more distinctive cases, we sought to determine individuals' knowledge of labels and the characteristics that associate or differentiate the types of CSR.

\section{Overview of the Studies}

We combined two mixed-method studies by integrating qualitative and quantitative data analysis - one using an association task and another based on free recall - to ascertain the clarity of the terms, plus a quantitative study to further characterize and differentiate CSR. In the first mixed-method study, in order to ascertain their knowledge of the CSRs, participants were asked to match the descriptions of four CSRs with their respective designations. The same was investigated in the second study, this time using an evocation task requesting that participants produce the terms by which the described CSRs are known. In the third study, participants were asked to clarify the descriptive and differentiating characteristics of three CSRs according to the presence of eight characteristics.

The criteria for participation were being between 18 and 29 years old and having Portuguese as their native language. The facilitating role of the university context for knowledge/experience of CSRs is well documented (Bogle, 2008), and we wanted to increase the likelihood of such knowledge by drawing mainly on university students. However, previous personal involvement in CSRs is not determinative of the knowledge that individuals have of the characteristics of different casual encounters (Wentland \& Reissing, 2014), showing that this knowledge is to some degree culturally-acquired, so personal experience of CSRs was not a condition for participation. Although previous sexual intercourse has been shown to increase individuals' knowledge of CSR labels (Wentland \& Reissing, 2014), this characteristic also was not made a condition for participation, because data collection would be by 
group and because in Portugal the average age of first experience of intercourse is approximately 16, constituting a normative experience (Reis et al., 2011).

At the end of the tasks in each of the three studies, the participants answered a sociodemographic questionnaire in writing or through an interview, providing information on age, gender, ethnicity, educational qualifications, religion, relational status, sexual orientation, whether they had already had sexual intercourse, number of partners, whether they had had casual sexual relationships, and the number of casual encounters. Sexual relationships were operationalized as oral, vaginal, and anal sex, and a defined CSR was presented. In all studies, the material was arranged in three versions so as to avoid order effects.

The studies were approved by the FPUL Ethics Committee, and participants gave their informed consent. There was no financial compensation for participating in the studies.

\section{Study 1}

\section{Method}

\section{Participants}

The study included 51 participants between 18 and 25 years old $(M=21.66, S D=$ 1.56), $65.8 \%$ female. The participants had had between 1 and 7 sexual partners in their lives so far, with the modal value being 1 partner. The number of casual sexual encounters indicated by the small number of participants who said they had already had casual sexual relationships varied between 1 and 20, with the modal value being two encounters (Table 1).

\section{Materials and Procedure}

To assess knowledge of CSR and the terms used to label them, we constructed a task that presented descriptions of four types of CSR encounter and four labels to designate these, with participants asked to match a label to each description. We chose to use the three clearest CSRs found in prior studies with Portuguese samples (Alvarez et al., 2019; Brandão, 2019; Silva, 2019) - friends with benefits, making out, and one night stand - plus booty call, an encounter infrequently mentioned in those studies but often found in the literature, which has different characteristics from the other three (Jonason et al., 2009). All labels were used in their Portuguese equivalents (amigos coloridos, curte, caso de uma noite), except booty call, which was found to be used mainly in English. The descriptions were drawn from the exploratory qualitative studies of the CSRs that Portuguese emerging adults know of or experience (e.g., Alvarez et al., 2019), with the description we found for booty call complemented by that of a study conducted for the same purpose with a Canadian sample (Wentland \& Reissing, 2011). The descriptions were checked by three young people to ensure their clarity (Appendix ). 
Table 1 Sociodemographic characteristics of the samples by study

\begin{tabular}{|c|c|c|c|}
\hline \multirow[t]{2}{*}{ Sociodemographic characteristics } & \multicolumn{3}{|l|}{ Participants $(\%) \dagger$} \\
\hline & Study $1(N=51)$ & Study $2(N=53)$ & Study $3(N=158)$ \\
\hline \multicolumn{4}{|l|}{ Relational status } \\
\hline No relationship & $16(42.1)$ & $17(51.5)$ & $70(52.2)$ \\
\hline Dating & $21(55.3)$ & $15(45.5)$ & $61(45.6)$ \\
\hline Other & $1(2.6)$ & $1(3.0)$ & $3(2.2)$ \\
\hline \multicolumn{4}{|l|}{ Ethnicity } \\
\hline White & $32(84.2)$ & $31(91.2)$ & $127(94.8)$ \\
\hline African & $6(15.8)$ & $2(5.9)$ & $3(2.2)$ \\
\hline Other & - & $1(2.9)$ & $4(3.0)$ \\
\hline \multicolumn{4}{|l|}{ Religion } \\
\hline Yes & $7(53.8)$ & $4(28.6)$ & $51(37.5)$ \\
\hline No & $6(46.2)$ & $10(71.4)$ & $85(62.5)$ \\
\hline \multicolumn{4}{|l|}{ Which? } \\
\hline Catholic & $5(71.4)$ & $3(75.0)$ & $44(88.0)$ \\
\hline Other & $2(28.6)$ & $1(25.0)$ & $6(12.0)$ \\
\hline \multicolumn{4}{|l|}{ Educational qualifications } \\
\hline Highersecondary education & $15(41.7)$ & $14(40.0)$ & $57(42.5)$ \\
\hline Until 3 years of universityeducation & $14(38.9)$ & $14(40.0)$ & $33(24.6)$ \\
\hline Undergraduate degree & $5(13.9)$ & $4(11.4)$ & $35(26.1)$ \\
\hline Master's degree & $2(5.6)$ & $3(8.6)$ & $9(22.9)$ \\
\hline \multicolumn{4}{|l|}{ Sexual orientation } \\
\hline Homosexual & - & - & $8(7.0)$ \\
\hline Bisexual & - & $2(16.6)$ & $8(7.0)$ \\
\hline Heterosexual & $12(100)$ & $12(83.3)$ & $98(86.0)$ \\
\hline \multicolumn{4}{|l|}{ Sexual intercourse } \\
\hline Yes & $10(83.3)$ & $12(85.7)$ & $111(82.2)$ \\
\hline No & $2(16.7)$ & $2(14.3)$ & $24(17.8)$ \\
\hline \multicolumn{4}{|l|}{ Casual sexual relationships } \\
\hline Yes & $6(46.2)$ & $6(42.9)$ & $70(52.2)$ \\
\hline No & $7(53.8)$ & $8(57.1)$ & $64(47.8)$ \\
\hline
\end{tabular}

Sociodemographic data are related to 38,35, and 145 participants in Studies 1, 2 and 3 respectively because we were not able to pair the data of 44 participants with the studies. This was due to some collection having taken place online due to the confinement required by the Covid-19 pandemic, including certain questions we had chosen not to ask in person because they could have embarrassed the participants.

The participants were instructed to match each description with a given designation, and they were asked whether they had used the process of elimination for any of them. The data were collected in person by paper-and-pencil, as well as face-toface online via Skype with the support of a master's student, with 26 participants completing the task in paper-and-pencil and 25 face-to-face online. The need to collect data online resulted from the study still being in progress when mandatory 
confinement was decreed during the Covid-19 pandemic. Individuals reporting details about their sexual behaviors to an interviewer are more likely to engage in impression management than those completing a paper-and-pencil questionnaire (McCallum \& Peterson, 2012). Some sociodemographic data were therefore not requested during the face-to-face collection (e.g., religion, sexual orientation, having already had sex, number of partners, having already had a CSR and number of casual sexual encounters) because these were considered to be more intimate questions that participants might have difficulty sharing in person.

\section{Analysis Procedures}

The analysis of the number of correct answers and mistakes in the associations between encounters and designations was performed through binary logistic regression. We categorized responses into a binary variable $(0=$ error; $1=$ hit $)$ and used this as a dependent variable regressed in the type of CSR used as a stimulus (friends with benefits vs. making out vs. one night stand vs. booty call), taking the effect of gender and age into account, seeking to minimize type I error as well as decisions based on spurious relationships. Age was transformed into a categorical variable from a cutoff point of $50 \%$ of the cases for each category $(18-21,>21$ years).

Subsequently, we performed an error analysis for CSR to find the types of CSR that produced the greatest number of errors. For this purpose, chi-square tests were used for each type of CSR. In instances that violated the Chi-square test assumption of having fewer than $20 \%$ of cells with an expected frequency under five, we used the Fisher Exact Test to check the significance of the results.

\section{Results}

The majority of participants correctly identified the labels corresponding to the CSR descriptions - friends with benefits (88.2\%), making out (84.3\%), one night stand (100\%), and booty call (88.2\%) - with no differences in the knowledge of the labels for the various CSRs (Table 2). However, of the 10 participants who said they had made associations by process of elimination, $9 / 13$ were less certain about booty call (2/13 for making out and 2/13 for friends with benefits). Age proved to be related to the ability to correctly identify labels with descriptions, with the younger participants making more correct associations than the older ones, with no gender differences (Table 2).

Analysis of the incorrect pairings showed that the number was not different among the various types of CSR - for friends with benefits, $\chi 2(1)=.667, p=.414$; for making out, $\chi^{2}(1)=.143, p=.705$, and for booty call, $\chi^{2}(1)=.667, p=.414$ - with such errors occurring very infrequently (19 errors in 204 responses, $9.3 \%$ ). 
Table 2 Logistic regression analysis of the associations between descriptions of four CSR and corresponding labels, Study $1(N=51)$

\begin{tabular}{lllllll}
\hline Predictor & $\beta$ & $S E \beta$ & Wald's $\chi^{2}$ & $d f$ & $p$ & $\mathrm{e} \beta$ (odds ratio) \\
\hline Constant & 3.718 & 1.188 & 9.792 & 1 & .002 & 41.186 \\
FWB & .000 & .693 & .000 & 1 & 1.000 & 1.000 \\
MO & -420 & .653 & .413 & 1 & .520 & .657 \\
ONS & 19.312 & 6353.05 & .000 & 1 & .998 & 243889752.4 \\
Age $(1=18-21,2>21)$ & -1.211 & .611 & 3.933 & 1 & .047 & .298 \\
Gender $(0=$ women, $1=$ man) & .519 & .622 & .695 & 1 & .404 & 1.680 \\
Test & & & $\chi^{2}$ & $d f$ & $p$ & \\
Overall model evaluation & & & & & & \\
$\mathrm{R}^{2}$ & & & 16.57 & 5 & .005 & \\
\hline
\end{tabular}

Outcome $(0=$ error, $1=$ correct); FWB (Friends with Benefits); MO (Making Out); ONS (One Night Stand); BC (Booty Call) as reference category.

\section{Discussion}

The majority of our participants (90\%) correctly associated each type of CSR encounter with its respective label, demonstrating that the CSR descriptions are recognized in the labels provided. These results are similar to those from Wentland and Reissing (2014), who validated the terms by having participants match the definitions of the main CSRs to their labels, with the majority accurately identifying the corresponding labels. Also, the fact that there was no predominance of errors associated with the types of CSR in our study shows that there is shared knowledge about these CSRs.

While all the types of encounter were correctly paired by more than $84 \%$ of participants, participants tended to resort to the process of elimination for the type that was not identified by a set of characteristics or a specific label in our previous studies - the booty call. We prefer to examine this possibility rather than the alternative "I don't know", since, due to the task involving only four terms (only one of which was less well-known), it would be easy to find an answer for the last one. Does this mean that booty calls do not exist in the experience of these young people? Behavior of this type most likely occurs, especially since these encounters are sometimes described (Brandão, 2019; Silva, 2019), but their characteristics are not clear enough to distinguish them from other CSRs so they are not assigned a specific label. The fact that we used the booty call encounter, which had scarcely been found in the conceptualization of CSRs in previous studies with Portuguese samples, served as a kind of check that helped us conclude that the participants recognized the terms for particular CSR encounters in the descriptions we chose for them.

Results of the matching were not sensitive to gender. Such differences could have been anticipated based on the slightly greater participation in casual sex by men, according to Peterson \& Hyde (2010) - but this meta-analysis also called attention to smaller gender differences in sexuality in societies with greater gender equality. It is not surprising that the relevance of gender in CSR has been inconclusive in the 
literature, since some studies have found that men engage in more casual sex than women (e.g., Luff, 2014; Grello et al., 2006) while these differences were nonexistent in other investigations (Correa et al., 2017; Garcia \& Reiber, 2008; Owen et al., 2010). As far as age is concerned, the greater knowledge on the part of the younger participants may owe to a greater perception of the nuances these CRS contain, such as that when certain features vary - namely, repetition of the encounters, emotional bonding between partners, and exchanges other than sexual ones - a progression between CSRs, and even from casual to committed relationships, can occur (e.g., Bogle, 2008). The ability to differentiate among them is of value in helping individuals to understand what to expect and how to act in these various encounters, and the greater insight shown by the younger participants may result from these encounters being more prevalent during the first years of university (e.g., Wade, 2017).

However, this study has some drawbacks, as the task is relatively simple and may not provide the clearest possible picture of the knowledge of the characteristics of the CSRs and their labels. For this reason, in Study 2, we used a free recall task, which is known to be more cognitively-demanding (Mulligan, 1997).

\section{Study 2}

\section{Method}

\section{Participants}

Fifty-three participants participated in this study, aged between 19 and 27 years $(M$ $=21.63, S D=1.52), 51.4 \%$ male. The range of sexual partners was 1 to 8 throughout life and the mode was 1 to 2 partners. Regarding the number of casual sexual encounters to which a small number of participants responded, this varied from two to 10 throughout life, with a modal value of two (Table 1).

\section{Materials and Procedure}

The written descriptions of the CSR were identical to those used in Study 1; instead of presenting the terms, blank spaces were given for the participants to write the name by which each encounter is known.

The data were collected in person through paper-and-pencil and online, the latter via Skype with the support of a master's student, with 32 participants completing the task in person and 21 online. The reasons for online collection were the same as those described for Study 1.

\section{Analysis Procedures}

The same analysis procedures were used as those described in Study 1 . 


\section{Results}

In response to our descriptions of the four CSRs, participants provided 35 different labels. The labels were $71.7 \%$ matching for the description of friends with benefits, $59.6 \%$ for that of making out, $66 \%$ for one night stand and $28.3 \%$ for booty call. The other terms were very diverse, with $65.7 \%$ of them only being used once to label a CSR description (Supplementary file). The degree to which the names were correctly provided proved to be different across the types of CSR; this was due to the greater difficulty in producing the term booty call compared to all other encounters. Neither age nor gender affected these results (Table 3 ).

Results of the analysis of the incorrect labels produced by participants were different for the various CSR types, $\chi^{2}(12)=41.69, p=.000$, Fisher $=38.09, p=$ .000 . Participants were less able to produce booty call, providing a greater range of alternative designations than were given for other encounters. Differences also arise from one night stand being mislabeled as making out more than by other terms.

Table 3 Logistic regression analysis of the labels produced for the descriptions of the four CSR, Study 2 $(N=53)$

\begin{tabular}{|c|c|c|c|c|c|c|}
\hline Predictor & $\beta$ & $S E \beta$ & Wald's $\chi^{2}$ & $d f$ & $p$ & $\mathrm{E} \beta$ (odds ratio) \\
\hline \multirow[t]{4}{*}{ Constant } & $1.039^{\mathrm{a}}$ & $.711^{\mathrm{a}}$ & $-2.134^{\mathrm{a}}$ & 1 & $.144^{\mathrm{a}}$ & $2.827^{\mathrm{a}}$ \\
\hline & $.268^{\mathrm{b}}$ & $.691^{\mathrm{b}}$ & $.150^{\mathrm{b}}$ & 1 & $.698^{\mathrm{b}}$ & $1.307^{\mathrm{b}}$ \\
\hline & $.592^{\mathrm{c}}$ & $.692^{\mathrm{c}}$ & $.732^{\mathrm{c}}$ & 1 & $.392^{\mathrm{c}}$ & $1.808^{\mathrm{c}}$ \\
\hline & $-1.305^{\mathrm{d}}$ & $.714^{\mathrm{d}}$ & $3.343^{\mathrm{d}}$ & 1 & $.067^{\mathrm{d}}$ & $.271^{\mathrm{d}}$ \\
\hline \multirow[t]{3}{*}{ FWB } & $.772^{\mathrm{b}}$ & $.543^{\mathrm{b}}$ & $2.019^{\mathrm{b}}$ & 1 & $.155^{\mathrm{b}}$ & $2.163^{\mathrm{b}}$ \\
\hline & $.447^{\mathrm{c}}$ & $.550^{\mathrm{c}}$ & $.662^{\mathrm{c}}$ & 1 & $.416^{\mathrm{c}}$ & $1.564^{\mathrm{c}}$ \\
\hline & $2.345^{\mathrm{d}}$ & $.569^{\mathrm{d}}$ & $16.958^{\mathrm{d}}$ & 1 & $.000^{\mathrm{d}}$ & $10.428^{\mathrm{a}}$ \\
\hline \multirow[t]{3}{*}{ MO } & $-772^{\mathrm{a}}$ & $.543^{\mathrm{a}}$ & $2.019^{\mathrm{a}}$ & 1 & $.155^{\mathrm{a}}$ & $.462^{\mathrm{a}}$ \\
\hline & $-.324^{c}$ & $.515^{\mathrm{c}}$ & $.397^{\mathrm{c}}$ & 1 & $.529^{\mathrm{c}}$ & $.723^{\mathrm{c}}$ \\
\hline & $1.573^{\mathrm{d}}$ & $.532^{\mathrm{d}}$ & $8.730^{\mathrm{d}}$ & 1 & $.003^{\mathrm{d}}$ & $4.820^{\mathrm{d}}$ \\
\hline \multirow[t]{3}{*}{ ONS } & $-447^{a}$ & $.550^{\mathrm{a}}$ & $.662^{\mathrm{a}}$ & 1 & $.416^{\mathrm{a}}$ & .639 \\
\hline & $.324^{\mathrm{b}}$ & $.515^{\mathrm{b}}$ & $.397^{\mathrm{b}}$ & 1 & $.529^{\mathrm{b}}$ & $1.383^{\mathrm{b}}$ \\
\hline & $1.897^{\mathrm{d}}$ & $.541^{\mathrm{d}}$ & $12.277^{\mathrm{d}}$ & 1 & $.000^{\mathrm{d}}$ & $6.667^{\mathrm{d}}$ \\
\hline \multirow[t]{3}{*}{ BT } & $-2.345^{\mathrm{a}}$ & $.569^{\mathrm{a}}$ & $16.958^{\mathrm{a}}$ & 1 & $.000^{\mathrm{a}}$ & $.096^{\mathrm{a}}$ \\
\hline & $-1.573^{\mathrm{b}}$ & $.532^{\mathrm{b}}$ & $8.730^{\mathrm{b}}$ & 1 & $.003^{\mathrm{b}}$ & $.207^{\mathrm{b}}$ \\
\hline & $-1.897^{\mathrm{c}}$ & $.541^{\mathrm{c}}$ & $12.277^{\mathrm{c}}$ & 1 & $.000^{\mathrm{c}}$ & $.150^{\mathrm{c}}$ \\
\hline Age $(1=18-21,2>21)$ & .364 & .382 & .910 & 1 & .340 & 1.440 \\
\hline Gender $(0=$ women, $1=\operatorname{man})$ & -639 & .384 & 2.766 & 1 & .096 & .528 \\
\hline Test & & & $\chi^{2}$ & $d f$ & $p$ & \\
\hline \multicolumn{7}{|l|}{ Overall model evaluation } \\
\hline $\mathrm{R}^{2}$ & & & 26.14 & 5 & .000 & \\
\hline
\end{tabular}

Outcome $(0=$ error, $1=$ correct); FWB (Friends with Benefits); MO (Making Out); ONS (One Night Stand); BC (Booty Call). ${ }^{\mathrm{a}} \mathrm{FWB}$ as reference category; ${ }^{\mathrm{b}} \mathrm{MO} ;{ }^{\mathrm{c}} \mathrm{ONS} ;{ }^{\mathrm{d}} \mathrm{BT}$ 


\section{Discussion}

Evocation tasks such as free recall are cognitively more demanding than tasks of mere association because they require intentional retrieval (Mulligan, 1997) and so may be a more robust indicator by which to make conclusions about the knowledge held by individuals. This study shows that, when faced with an evocation task, knowledge of labels is more limited (56\%). The greater difficulty exhibited in the designation of booty calls and the existence of more inaccuracies in the labeling of this type of encounter, which were expected, points to the importance of qualitative studies for determining how participants conceptualize CSR as well as the importance of ascertaining the terms used to label them. Types of encounters that are poorly-defined or that lack a label to identify them will not be well-organized in a script and, therefore, will be little used for the recognition of specific information, construction of its meaning, and orientation for action (Simon \& Gagnon, 1986). Such categories will therefore fail as measures for social and cognitive economy. The diversity of the terms found for naming the four CSRs and the fact that a higher proportion of the designations offered were in English (16\%), especially for booty call, point to somewhat limited cultural knowledge of the terms for these CSRs and to their meaning being not yet widely established.

Gender continued not to be a differentiating factor for knowledge of the labels, and, contrary to what was found in Study 1, age was no longer a differentiating factor. We speculate that this more-demanding task required having clear typifications of the CSRs already available/constructed through the language, with intersubjective knowledge of these types being no greater among the youngest than among the oldest, which may be an indicator that no significant changes in the social knowledge of CSRs have occurred over the last 10 years.

This study highlights three encounters for which there are clear-cut terms, and the existence of these labels brings greater certainty that the encounters relate to social realities well known by the participants. However, although the clearer CSRs point to some diversity in the encounters, this does not prove that they are significantly different from each other. This is all the more important to keep in mind as the boundaries between them are diffuse (Claxton \& van Dulmen, 2013). Although CSRs are relationships that vary according to the factors of time, emotion, sexual behavior, and acquaintance (Wentland \& Reissing, 2011), the quantitative and nondescriptive investigation of these characteristics has yet to be accomplished. With the clearer CSR types identified in this study, examining the similarities and differences between them would increase the likelihood of building on known and meaningful realities for participants. 


\section{Study 3}

\section{Method}

\section{Participants}

This study involved 158 participants aged between ages 18 and 29 ( $M=20.61, S D$ $=1.55), 73.5 \%$ of whom were women. The number of sexual partners in this sample varied between 1 and 30 over life so far, with a modal value of 1 . As for the number of casual sexual encounters, this varied between 0 and 28 , with a modal value of one (Table 1).

\section{Materials and Procedure}

The three most-clearly understood encounters in Studies 1 and 2 (friends with benefits, making out, one night stand) were listed, each with seven descriptive characteristics found in previous studies (e.g., Alvarez et al., 2019); booty call was not used due to the lack of evidence for a shared understanding of the term's characteristics in those studies. Table 4 shows these characteristics, to which we have added another: sexual protection in the form of condom use. Participants were asked to comment on the presence of each characteristic in these relationships, using a seven-point semantic differential between -3 (unequivocal absence of the characteristic) and +3 (unequivocal presence of the characteristic).

The data were collected in person and online, the latter via Zoom, with 52 participants completing the task in person and 106 online. The reasons for online collection are described in Study 1.

\section{Analysis Procedures}

A preliminary analysis showed the multivariate residuals to be normally distributed. Thus, the multivariate analysis of variance (MANOVA) was used to ascertain the presence of each characteristic in the CSR, and the significant results were interpreted on the basis of an ANOVA with a Fisher's Least Significant Difference posthoc test.

A complementary analysis was carried out to analyze the differences in the characteristics of the CSR with gender and age as covariates (MANOVA). Age was transformed into a categorical variable from a cutoff point of $50 \%$ of the cases for each category (18-20, > 20 years). 


\section{Results}

The sexual characteristics - sexual involvement and protection - were those most present in the CSRs, and commitment and exclusivity were those that least characterized these encounters, and emotional involvement had an average value close to zero, defined as a neutral opinion regarding the presence/absence of the characteristic in question (Table 4).

We found a significant multivariate effect of CSRs on the characteristics by which they are defined, $F(16,590)=20.26, p=.000, \eta_{\mathrm{p}}{ }^{2}=.355$. Univariate effects (ANOVAs) revealed significant effects of the CSRs on almost all variables: Emotional involvement $\left(F(2,301)=99.81, p=.000, \eta_{\mathrm{p}}{ }^{2}=.399\right)$; Sexual involvement $\left(F(2,301)=9.19, p=.000, \eta_{\mathrm{p}}{ }^{2}=.058\right)$; Repetition $(F(2,301)=111.98, p=$ $\left..000, \eta_{\mathrm{p}}{ }^{2}=.427\right)$; Commitment $\left(F(2,301)=96.35, p=.000, \eta_{\mathrm{p}}{ }^{2}=.390\right)$; Exclusivity $\left(F(2,301)=54.06, p=.000, \eta_{\mathrm{p}}{ }^{2}=.264\right)$; Premeditation $(F(2,301)=55.52$, $\left.p=.000, \eta_{\mathrm{p}}{ }^{2}=.269\right)$; Partner acquaintance $\left(F(2,301)=138.64, p=.000, \eta_{\mathrm{p}}{ }^{2}=\right.$ .479). Only Sexual protection (condom use) did not show a significant effect $\left(F(2,301)=.11, p=.893, \eta_{\mathrm{p}}^{2}=.001\right)$.

Post-hoc analysis showed that the CSR were all distinguished from each other in all significant characteristics. The significant characteristics were more present in friends with benefits, followed by making out and one night stand, except in sexual involvement, which characterized making out less than the other two encounters (Table 4).

No gender differences were found for any characteristic of the $\operatorname{CSR}(F(8,289)=$ $\left..65, p=.739, \eta_{\mathrm{p}}{ }^{2}=.018\right)$. A main effect was found for categorical age as covariable, $F(8,289)=2.69, p=.007, \eta_{\mathrm{p}}{ }^{2}=.069$. Univariate effects (ANOVAs) revealed significant effects of age on two characteristics of the encounters: Sexual involvement

Table 4 Mean scores and (standard deviation) of characteristics defining each CSR, study $3(N=158)$

\begin{tabular}{|c|c|c|c|c|c|c|c|c|}
\hline & $\begin{array}{l}\text { Friends With } \\
\text { Benefits }(n= \\
108)\end{array}$ & & $\begin{array}{l}\text { Making } \\
\text { Out }(n= \\
98)\end{array}$ & & $\begin{array}{l}\text { One-Night } \\
\text { Stand }(n= \\
98)\end{array}$ & & Total Mean & \\
\hline $\begin{array}{l}\text { Emotional } \\
\text { involvement }\end{array}$ & $1.26^{\mathrm{a}}$ & (1.44) & $.22^{\mathrm{b}}$ & $(1.65)$ & $-1.76^{\mathrm{c}}$ & $(1.55)$ & -046 & (1.99) \\
\hline $\begin{array}{l}\text { Sexual } \\
\text { involvement }\end{array}$ & $1.69^{\mathrm{a}}$ & (1.54) & $.69^{\mathrm{c}}$ & $(1.53)$ & $1.22^{\mathrm{b}}$ & (1.93) & 1.22 & $(1.72)$ \\
\hline Repetition & $1.68^{\mathrm{a}}$ & $(1.65)$ & $.74^{\mathrm{b}}$ & (1.54) & $-1.67^{\mathrm{c}}$ & (1.74) & .30 & $(2.16)$ \\
\hline Commitment & $.43^{\mathrm{a}}$ & (1.84) & $-.56^{\mathrm{b}}$ & $(1.76)$ & $-2.58^{c}$ & $(.93)$ & -.86 & $(2.01)$ \\
\hline Exclusivity & $.36^{\mathrm{a}}$ & $(1.90)$ & $-.33^{\mathrm{b}}$ & (1.88) & $-2.17^{\mathrm{c}}$ & $(1.57)$ & -.68 & $(2.08)$ \\
\hline Premeditation & $1.49^{\mathrm{a}}$ & $(1.51)$ & $.49^{\mathrm{b}}$ & (1.65) & $-.98^{\mathrm{c}}$ & (1.89) & .37 & (1.97) \\
\hline $\begin{array}{l}\text { Sexual protec- } \\
\text { tion }\end{array}$ & 1.99 & $(1.56)$ & 1.90 & (1.71) & 1.89 & (1.91) & 1.93 & $(1.72)$ \\
\hline $\begin{array}{l}\text { Partner } \\
\text { acquaintance }\end{array}$ & $1.94^{\mathrm{a}}$ & $(1.44)$ & $.51^{\mathrm{b}}$ & (1.69) & $-1.70^{\mathrm{c}}$ & (1.59) & .30 & $(2.17)$ \\
\hline
\end{tabular}

In each section of lines, the means that do not share the same superscript represent significant differences according to the LSD test, $p<.001$, except between Friends with Benefits and One-Night Stand in Sexual Involvement $(p<.05)$. 
$\left(F(1,301)=20.76, p=.006, \eta_{\mathrm{p}}{ }^{2}=.025\right)$, higher in older participants $\left(M_{18-20}=.97\right.$, $\left.S D=1.82 ; M_{>20}=1.50, S D=1.55\right)$ and Exclusivity $(F(1,301)=23.81, p=.006$, $\left.\eta_{\mathrm{p}}{ }^{2}=.025\right)$, lower in older participants $\left(M_{18-20}=-.38, S D=2.12 ; M_{>20}=-1.01, S D\right.$ $=2.00)$.

\section{Discussion}

This study provides quantitative evidence of the distinctiveness of the types of CSR, as suggested in qualitative literature (e.g., Wentland \& Reissing, 2011, 2014), based on sexual and emotional involvement and differentiated also by commitment, exclusivity, repetition, premeditation of the encounter, and partner acquaintance. Although the sexual characteristics are those most unequivocally present in the CSRs, all the characteristics contributed to the distinctions participants drew between the types of encounter, with the exception of sexual protection, which they judged to be equally present in all encounters. These findings help validate a more nuanced perspective on the psychoemotional, behavioral, and sexual characteristics in the CSRs among emerging adults.

Perhaps it is too affirmative to say that some characteristics contradict the stereotype of casual sex and get closer to what is expected in long-term relationships, but in this study emotional involvement, commitment, and exclusivity are far from being unmistakably absent in CSR, with friends with benefits tending more to their presence than absence. The complexity and the hybrid character of CSRs has been shown in their relation to committed traditional romantic relationships in affectionate (e.g., Garcia et al., 2018), sexual (Furman \& Shaffer, 2011), and emotional and sexual behaviors (Jonason et al., 2011). We were able to show this in several characteristics and in a wider variety of CSRs, the clearest cases in Portuguese samples.

As has been the case for various casual sexual behaviors (e.g., Correa et al., 2017), we found no gender differences in the characteristics attributed to these encounters, although the gender imbalance in the sample should be taken into account. The sensitivity of some characteristics of the types of encounter to age - making their sexual realization more frequent and less exclusive - indicates the role experience plays in how these encounters are conceptualized; this makes their investigation relevant in adults, a little-studied population.

The manifestation of more differences than similarities between types of encounters indicates the pertinence of variations in behavioral (e.g., sexual practices, sexual protection) and in emotional aspects (e.g., expectations) for efforts to promote sexual health. The rates of condom use in CSR are contradictory - in some cases the rate does not exceed 50\% (e.g., Lewis et al., 2012; Reece et al., 2010), while in other cases they are used by the majority, although non-use remains nontrivial (Bearak, 2014). We speculate, therefore, that their clear presence in the CSR types and the similarity found regarding their use may be an instance of social desirability bias, as already detected in previous studies, in which condom use was indicated by self-reporting but absent when studied through less-deliberative responses based on 
the memory of actions present in sexual scripts (Alvarez \& Garcia-Marques, 2008). This matter requires closer examination in future investigations.

\section{General Discussion}

The terminology, definitions, and categories used in the studies, insofar as they reflect participants' own conceptualizations of the types of CSR, improve response consistency and the accuracy of research. We wanted to ascertain what knowledge emerging adults have of the types of CSR and of the most clearly-defined terms that are used, as well as whether the different casual experiences were perceived as distinct categories of CSR. The definitions, labels, and characteristics of the CSRs used in this study, based on previous studies among Portuguese emergent adults (Alvarez et al., 2019), permit us to conclude that three types of CSR - friends with benefits, one-night stand - and, to a lesser extent, making out - may be used in future research as these are understood and associated with consensual labels. In addition, these CSRs proved to be distinct, as they are more different than similar in terms of a set of psycho-emotional, behavioral, and sexual characteristics by which participants distinguished among them when evaluated jointly.

Did our participants correctly match the given labels to the descriptions of CSRs, and were these matches sensitive to gender and age? The majority of the participants were able to match the description to each of the CSR labels - friends with benefits, making out, one-night stand, and booty call (the later scarcely found in our previous research). These results are in line with others where a correct match is considered evidence of the recognition of a shared understanding of CSR archetypes and of the nuances that distinguish them (Wentland \& Reissing, 2014). That participants resorted to the process of elimination only for matching booty call is an indicator that participants had the culturally-acquired knowledge to understand and recognize the other CSRs. What we found regarding the types of main CSR and the role of gender differed from the few other studies that exist (Wentland \& Reissing, 2011, 2014), in which the women's greater ability to identify CSR labels was attributed to the costs of engaging in casual sexual activity; this was perhaps compensated for in our study by the fact of men and women having equal experience, as identified in research (e.g., Correa et al., 2017; Owen et al., 2010), reducing the cost gap between genders. In addition, higher familiarity with the CSRs on the part of participants younger than 21 years old, which we inferred from their making more correct matches, may be an indication of their greater pertinence in recent years. With this study, we add to the confidence about which labels or cultural scenarios of CSR this sample has enough familiarity with, as well as what underlies each, increasing research validity on casual sex in the Portuguese cultural context when using these labels.

Did these results persist when participants were not supplied with the labels, but asked to produce them? This proved to be a more difficult task than the simple association, but the majority of the participants were able to succeed. The exception was the CSR seeking the label booty call, which was only correctly produced by a quarter of participants; this was integrated as a check because it was not found in our 
previous research as one of the terms most used to describe, think about, and name a CSR (Alvarez et al., 2019). Gender did not have an effect on the production of labels, nor did age, which may be another indicator of recent changes in the sociosexual setting, such that these changes have not yet been sufficiently established and shared. We are unaware of similar research, and we believe that the study of labels by free recall, due to the higher cognitive demand of this task, is likely to give a more reliable picture of the existing knowledge about CSR. This study draws attention to the fact that the use of relevant examples of CSR is culturally-specific. Clearcut terms are more likely to be associated with scripted information (Rosch, 1978), which is used by individuals to understand sexual situations and guide their actions, so they are useful for studying sexual scripts.

Were friends with benefits, making out, and one-night stand perceived as distinct or overlapping categories of CSR, and was this affected by gender and age? The types of CSR differed in all the psycho-emotional, behavioral, and sexual characteristics used to evaluate them (sexual and emotional involvement, repetition, commitment, exclusivity, premeditation, and partner acquaintance), except for condom use. All these elements characterize friends with benefits, are partially present in making out, without commitment or exclusivity, and are almost absent in the one night stand, except for the presence of sexual involvement and protection. These heterogeneous patterns contribute to validate a more nuanced perspective of CSRs among emerging adults. Gender does not differentiate the distinctiveness of CSRs for individuals, but differences by age indicate that CSRs perform different functions along emerging adulthood, with sexual realization and less exclusivity being perceived more by those on the older end of our sample, perhaps indicating that CSRs are progressively less focused around the learning of sexual interaction and of mating strategies and understood more realistically, based on what is found in the literature about higher levels of concurrent CSRs (Paik, 2010).

The existence of a diversity of CSRs has long been highlighted (e.g., Claxton \& van Dulmen, 2013; Garcia et al., 2012; Wentland \& Reissing, 2011) but this was an assumption in need of nondescriptive validation (Claxton \& van Dulmen, 2013). These findings empirically confirm the value of differentiating among types of CSR in research, and they provide a basis for further validation studies with larger samples. The high use of condoms in all types of CSR, which did not differ among them, may be a very positive result; on the other hand, we should perhaps suspect that this result is skewed by social desirability bias, given that most characteristics, namely partner-acquaintance, premeditation of the encounters (e.g., Weaver et al., 2011), and emotional involvement (e.g., Misovich et al., 1997), influence planning for safe sex and negotiation for condom use, and these showed differences among the types of CSR we examined. Therefore, we suggest that condom use will also vary among them, something that remains to be investigated.

There are a number of limitations that should be noted. The use of convenience samples requires caution when seeking to generalize the findings. In addition, participants were mainly White, college students, heterosexual, and self-selected, with more women in two of the studies. This may have decreased our ability to detect gender differences that might otherwise be significant. Volunteer bias characterized by more sexually-experienced and permissive participants (e.g., Wiederman, 1999) 
might have meant that this sample had higher-than-usual knowledge of the terminology and the distinguishing features of the CSR types. Second, the use of one label (booty call) in English, due to its being preferred in this language in former samples, may have created additional difficulty in the study of free recall of the labels. Additionally, caution should also be taken with regard to the understanding of making out, as $40 \%$ of participants attributed another label to the description of the encounter. Third, as far as sexual experience is concerned, due to incomplete data on "having had sex", it was not possible to draw conclusions about the role of this variable in the different studies; the same is the case concerning whether participants had prior casual sexual experience, which was not required for participation in the studies as it has presented no influence on ability to identify the labels for types of CSR (Wentland \& Reissing, 2014), but whose influence regarding the distinctiveness of the CSRs remains to be investigated. Fourth, there may be other CSRs with clearcut terms and defining characteristics that we may not have captured in previous research, and so we could have extended the number of less-familiar CSRs in Studies 1 and 2 beyond the booty call. This limitation extends to the characteristics used in Study 3. We wonder whether using characteristics related to romantic relationships (e.g., passion and intimacy, which are less present in CSRs, and characteristics related to sexual behavior, which are more present [Rodrigue et al., 2018]) would have further distinguished the types of CSR from each other. In addition, by analogy with hedonic contrast being found to be greater for stimuli within a common category than for those from separate categories (Zellner et al., 2003), we wonder whether the use of CSR alone, rather than including traditional romantic relationships, could have increased the differences between the types of CSR.

Having found clear-cut terms for specific descriptions and differences in the characteristics of CSRs when studied jointly, we may emphasize the need to specify the particular type of CSR under examination when research is conducted in this area. This is all the more important as the diversity of labels in use leaves open the possibility of still-imprecise meanings and limits to intersubjectivity regarding CSRs.

With this study we are provided with more robust arguments to be deployed in investigating the types of CSR that have been found, their labels, and their definitions. These terms have now been shown to be descriptors of the definitions not only in an association task, but also by evocation, and the CSRs have been shown to have distinct characteristics. Because there is a greater probability of sexual scripts existing for these CSRs, these being the types of encounters that are recognized, distinctive, and understood by the participants, the use of these terms in research is optimal for further understanding the sexual behavior and how to act in the CSRs.

Some of the CSRs we found are among those most mentioned in the literature, such as friends with benefits and one-night stand. However, making out is also one of the main CSRs we found, and booty call is a type of CSR that does not seem to have a set of constitutive elements or clear designation shared by the individuals. Hence, the results show that the most well-known and distinctive CSRs vary among cultures, even though intercultural influences exist, as reflected in participants' use of English terms $16 \%$ of the time in our sample.

Knowledge of the diversity and differentiation between the types of encounter can also have implications for a healthier experience of CSRs. They can be used to 
increase communication between partners to better define the sexual situations in which they are involved, decreasing unwanted experiences. On the other hand, this knowledge shows us that some CSRs have characteristics that are usually attributed to romantic relationships, with implications for not using a condom, which is often associated with more affection, intimacy, and expectations of future commitment (Alvarez \& Garcia-Marques, 2011).

Moving beyond prior work, we have deepened knowledge of the labels and clear cases of CSRs through a more demanding free-recall study, rather than mere association, and have explored a set of terms for CSRs in conjunction to studying how they are distinct from each other. This investigation is not only useful for the study of CSR in Portugal, but is also one of the few studies that has validated the terms employed, and it contributes to a quantitative exploration of the distinction between several types of casual sexual relationship.

\section{Appendix}

\section{Friends With Benefits}

Relationship between two people who already have a previous friendship, with trust between the parties, but without romantic feelings involved. In this relationship, rules are established, and its termination is formal and discussed. It does not involve commitment, nor is it assumed.

\section{Making Out}

Spontaneous and less-planned encounter, which may occur between acquaintances or strangers. Rules are not established and penetrative sex is not expected (it involves only kissing, embracing, and caressing). This encounter may happen more than once with the same person.

\section{One-Night Stand}

A sexual encounter between strangers who will not see each other again, usually accompanied by the consumption of alcohol and/or drugs in night spaces, with attraction being the triggering factor of the relationship. Rules are not set, nor is there commitment. Communication between those involved is very limited and, therefore, termination does not require a formal conversation. 


\section{Booty Call}

Repeated encounters that may occur between strangers as well as acquaintances, in which the main objective is the satisfaction of sexual desire. If the meeting is satisfactory for both, contact is maintained. In these relationships, meetings are planned mainly through digital media (messages, calls, or social networks) after a mutual attraction.

Supplementary Information The online version contains supplementary material available at https://doi. org/10.1007/s12119-021-09859-0.

Acknowlegements The authors would like to thank Carina Silva for her contribution to the data collection.

Funding This study was supported by the Portuguese Science Foundation, FCT (Grant PTDC/ PSI-GER/28530/2017).

Availability of data and material Data are available upon request to the first author.

\section{Declarations}

Conflict of interest Maria-João Alvarez declares that she has no conflict of interest; Cícero R. Pereira declares he has no conflict of interest; Cristina A. Godinho declares she has no conflict of interest; Rita Luz declares she has no conflict of interest.

Ethical Approval All procedures performed in studies involving human participants were in accordance with the ethical standards of the institutional and/or national research committee and with the $1964 \mathrm{Hel}$ sinki declaration and its later amendments or comparable ethical standards.

Informed Consent Informed consent was obtained from all individual participants included in the study.

\section{References}

Afifi, W. A., \& Faulkner, S. L. (2000). On being 'just friends': The frequency and impact of sexual activity in cross-sex friendships. Journal of Social and Personal Relationships, 17, 205-222.

Alvarez, M. J. (2005). Representações cognitivas e comportamentos sexuais de risco: O guião e as teorias implícitas da personalidade nos comportamentos de protecção sexual [Cognitive Representations of Sexual Risk Behaviors: The Condom and the Implicit Theories of Personality in Sexual Protective Behaviors]. FCT/FCG.

Alvarez, M. J., \& Garcia-Marques, L. (2008). Condom inclusion in cognitive representations of sexual encounters. Journal of Sex Research, 45, 358-370.

Alvarez, M.-J., \& Garcia-Marques, L. (2011). Cognitive and contextual variables in sexual partner and relationship perception. Archives of Sexual Behavior, 40, 407-417.

Alvarez, M.-J., Garcia, M., \& Pereira, C. (2019). A diversidade dos relacionamentos casuais e suas características [The diversity of casual relationships and their characteristics]. Psicologia, 33(2), 9-26.

Alvarez, M. J., \& Nogueira, J. (2008). Como definem os estudantes ter sexo, parceiro sexual e infidelidade [How students define sex, sexual partner, and infidelity]. Psicologia, XXII, 1, 59-76.

Bearak, J. M. (2014). Casual contraception in casual sex: Life-cycle change in undergratuates' sexual behavior in hookups. Social Forces, 93, 483-513.

Berntson, M. A., Hoffman, K. L., \& Luff, T. L. (2014). College as context: Influences on interpersonal sexual scripts. Sexuality \& Culture, 18, 149-165. 
Bogle, L. (2008). Hooking up: Sex, dating, and relationships on campus. . New York University Press.

Brandão, R. (2019). Aprofundamento da diversidade dos relacionamentos sexuais casuais: Características e especificidades no género feminino [In-depth of the diversity of casual sexual relationships: Characteristics and specificities in female gender]. Lisboa: Faculdade de Psicologia, Universidade de Lisboa (Master thesis). https://repositorio.ul.pt/handle/10451/41545

Cantor, N. (1980). Perceptions of situations: Situation prototypes and person-situation prototypes. In D. Magnusson (Ed.), The situation: An interactional perspective. (pp. 229-244). Lawrence Erlbaum.

Claxton, S., \& van Dulmen, M. (2013). Casual sexual relationships and experiences in emerging adulthood. Emerging Adulthood, 1, 138-150.

Correa, A. B., Castro, A., Barrada, J. R., \& Ruiz-Gómez, P. (2017). Sociodemographic and psychosexual characteristics of students from a Spanish university who engage in casual sex. Sexuality Research \& Social Policy, 14, 445-453.

DeLamater, J. D., \& Hide, J. S. (1998). Essentialism vs. social constructionism in the study of human sexuality. Journal of Sex Research, 35, 10-18.

Furman, W., \& Shaffer, L. (2011). Romantic partners, friends, friends with benefits, and casual acquaintances as sexual partners. Journal of Sex Research, 48, 554-564.

Garcia, J. R., Gesselman, A. N., Massey, S. G., Sibold-Simpson, S. M., \& Merriwether, A. M. (2018). Intimacy through casual sex: Relational context of sexual activity and affectionate behaviours. Journal of Relationship Research, 9(e12), 1-10.

Garcia, J. R., \& Reiber, C. (2008). Hook-up behavior: A biopsychosocial perspective. Journal of Social, Evolutionary, and Cultural Psychology, 2, 192-208.

Garcia, J., Reiber, C., Massey, S., \& Merriwether, A. (2012). Sexual hookup culture: A review. Review of General Psychology, 16, 161-176.

Grello, C., Welsh, D., \& Harper, M. (2006). No strings attached: The nature of casual sex in college students. Journal of Sex Research, 43, 255-267.

Hatfield, E., Hutchison, E. S., Bensman, K., Young, D., \& Rapson, R. (2012). Cultural, social, and gender influences on casual sex: New developments. In J. M. Turn \& A. D. Mitchell (Eds.), Social psychology: New developments. (pp. 1-37). Nova Science.

Heldman, C., \& Wade, L. (2010). Hookup culture: Setting a new research agenda. Sexuality Research \& Social Policy, 7, 323-333.

Jonason, P. K. (2013). Four functions for four relationships: Consensus definitions of university students. Archives of Sexual Behavior, 42, 1407-1414.

Jonason, P. K., Li, N. P., \& Cason, M. J. (2009). The "booty call": A compromise betwen men's and women's ideal mating strategies. Journal of Sex Research, 46, 460-470.

Jonason, P. K., Li, N. P., \& Richardson, J. (2011). Positioning the booty-call relationship on the spectrum of relationships: Sexual but more emotional than one-night stand. Journal of Sex Research, 48, 486-495.

Klipfel, K. M., Claxton, S. E., \& van Dulmen, M. H. (2014). Interpersonal aggression victimization within casual sexual relationships and experiences. Journal of Interpersonal Violence, 29, 557-569.

Lewis, M. A., Granato, H., Blayney, J. A., Lostutter, T. W., \& Kilmer, J. R. (2012). Predictors of hooking up sexual behaviors and emotional reactions among U.S. college students. Archives of Sexual Behavior, 41, 1219-1229.

McCallum, E. B., \& Peterson, Z. D. (2012). Investigating the impact of inquiry mode on self-reported sexual behavior: Theoretical considerations and review of the literature. Journal of Sex Research, 49(2-3), 212-226.

Misovich, S., Fisher, J., \& Fisher, W. (1997). Close relationships elevated HIV risk behavior evidence and possible underlying psychological processes. Review of General Psychology, 1, 72-107.

Mongeau, P. A., Knight, K., Williams, J., Eden, J., \& Shaw, C. (2013). Identifying and explicating variation among friends with benefits relationships. Journal of Sex Research, 50, 37-47.

Mulligan, N. W. (1997). Attention and implicit memory tests: The effects of varying attentional loading $\mathrm{n}$ conceptual priming. Memory \& Cognition, 25, 11-17.

Nelson, L. E., Morrison-Beedy, D., Kearney, M. H., \& Dozier, A. (2011). Sexual partner type taxonomy use among urban black adolescent mothers in the United States. The Canadian Journal of Human Sexuality, 20(1-2), 1-10. 
Owen, J. J., Rhoades, G. K., Stanley, S. M., \& Fincham, F. D. (2010). "Hooking up" among college students: Demographic and psychosocial correlates. Archives of Sexual Behavior, 39, 653-663.

Paik, A. (2010). The contexts of sexual involvement and concurrent sexual partnerships. Perspectives on Sexual and Reproductive Health, 42, 33-42.

Petersen, J. L., \& Hyde, J. S. (2010). A meta-analytic review of research on gender differences in sexuality, 1993-2007. Psychological Bulletin, 136, 21-38.

Pitts, M., \& Rahman, Q. (2001). Which behaviors constitute "having sex" among university students in the UK? Archives of Sexual Behavior, 30, 169-176.

Reece, M., Herbenick, D., Schick, V., Sanders, S., Dodge, B., \& Fortenberry, D. (2010). Condom use rates in a national probability sample of males and females ages 14 to 94 in the United States. Journal of Sexual Medicine, 7(suppl 5), 266-276.

Regan, P. C., \& Dreyer, C. S. (1999). Lust? Love? Status? Young adults' motives for engaging in casual sex. Journal of Psychology and Human Sexuality, 11, 1-24.

Reis, M., Ramiro, L., Gaspar de Matos, M., \& Diniz, J. A. (2011) The effects of sex education in promoting sexual and reproductive health in Portuguese university students. Procedia - Social and Behavioral Sciences, 29: 477-485.

Richters, J., \& Song, A. (1999). Australian university students agree with Clinton's definition of sex. British Medical Journal, 318, 1011-1012.

Rifkin, A. (1985). Evidence for a basic level in event taxonomies. Memory \& Cognition, 13, 538-556.

Rodrigue, C., Blais, M., Lavoie, F., Adam, B. D., Goyer, M.-F., \& Magontier, C. (2018). Passion, intimacy, and commitment in casual sexual relationships in a Canadian sample of emerging adults. Journal of Sex Research, 55, 1192-1205.

Rodrigue, C., Blais, M., Lavoie, F., Adam, B. D., Magontier, C., \& Goyer, M.-F. (2015). The structure of casual sexual relationships and experiences among single adults aged 18-30 years old: A latent profile analysis. The Canadian Journal of Human Sexuality, 24(3), 215-227.

Rosch, E. (1978). Principles of categorization. In E. Rosch \& B. Lloyd (Eds.), Cognition and categorization. (pp. 27-48). Lawrence Erlbaum.

Rosch, E., \& Mervis, C. B. (1975). Family resemblances: Studies in the internal structure of categories. Cognitive Psychology, 7, 575-605.

Rosch, E., Mervis, C. B., Gray, W. D., Johnson, D. M., \& Boyes-Braem, P. (1976). Basic objects in natural categories. Cognitive Psychology, 8, 382-439.

Roth, E. M., \& Shoben, E. J. (1985). The effect of context on the structure of categories. Cognitive Psychology, 15, 346-378.

Silva, M. (2019). Aprofundamento da diversidade dos relacionamentos casuais e suas especificidades para o género masculino. [In-depth of the diversity of casual sexual relationships and their particularities in male gender.] Lisboa: Faculdade de Psicologia, Universidade de Lisboa (Master thesis). https://repositorio.ul.pt/handle/10451/41540

Simon, W., \& Gagnon, J. (1986). Sexual scripts: Permanence and change. Archives of Sexual Behavior, 15, 97-120.

Singer, M., Erikson, P., Badiane, L., Diaz, R., Ortiz, D., Abraham, T., \& Nicolaysen, A. (2006). Syndemics, sex, and the city: Understanding sexually transmitted diseases in social and cultural context. Social Science \& Medicine, 63, 2010-2021.

Swan, D., \& Thompson, S. (2016). Monogamy, the protective fallacy: Sexual versus emotional exclusivity and the implication for sexual health risk. Journal of Sex Research, 53, 64-73.

Wade, L. (2017). American hookup: The new culture of sex on campus. W. W. Norton.

Weaver, S., \& Herold, E. (2000). Casual sex and women. Journal of Psychology \& Human Sexuality, $12(3), 23-40$

Weaver, A. D., MacKeigan, K. L., \& MacDonald, H. A. (2011). Experiences and perceptions of young adults in friends with benefits relationships: A qualitative study. Canadian Journal of Human Sexuality, 20(1-2), 41-53.

Wentland, J., \& Reissing, E. (2011). Taking casual sex not too casually: Exploring definitions of casual sexual relationships. Canadian Journal of Human Sexuality, 20(3), 75-91.

Wentland, J., \& Reissing, E. (2014). Identifying definitions for one night stands, booty calls, fuck buddies, and friends with benefits. Canadian Journal of Human Sexuality, 23(3), 23-40. 
Wiederman, M. W. (1999). Volunteer bias in sexuality research using college student participants. Journal of Sex Research, 36, 59-66.

Wiederman, M. W. (2016). Sexual script theory: Past, present, and future. In J. DeLamater \& R. F. Plante (Eds.), Handbook of the sociology of sexualities. (pp. 7-22). Springer.

Zellner, D. A., Rohm, E. A., Bassetti, T. L., \& Parker, S. (2003). Compared to what? Effects of categorization on hedonic contrast. Psychonomic Bulletin \& Review, 10, 468-473.

Publisher's Note Springer Nature remains neutral with regard to jurisdictional claims in published maps and institutional affiliations. 